\title{
Comunicación
}

\section{Comparación de Dos Modelos de Pipetas de Inseminación Artificial en la Cateterización del Canal Cervical de la Oveja}

\author{
Comparison of Two Models of Artificial Insemination Pipettes in the \\ Catheterization of the Cervical Canal in SheeP
}

Francisco Rodríguez Gavancho ${ }^{1,3}$, Juan Muscari Greco ${ }^{1}$, Cesar Condori Chipana ${ }^{2}$

\section{Resumen}

El objetivo del presente trabajo fue comparar la profundidad de penetración de un colorante en el canal cervical de la oveja, simulando una inseminación con una pipeta de uso tradicional con otra de diseño especial que dispone un globo inflable en el extremo libre que impide el reflujo del colorante. Ochenta tractos reproductivos de ovejas vacías fueron recuperados de un camal en Lima, Perú, y distribuidos al azar para su cateterización con una de las dos pipetas, depositando $0.5 \mathrm{ml}$ de azul de metileno. El globo de la pipeta de diseño especial se infló antes del depósito del colorante. Se abrió cada una de las cérvix y parte del útero y se midió la profundidad de ingreso de las pipetas y del colorante, donde el borde del primer pliegue cervical se asignó como punto cero (0). Ambas pipetas penetraron $19.9 \pm 9.6 \mathrm{~mm}$. El colorante alcanzó la misma profundidad con la pipeta tradicional y $60.0 \pm 9.7 \mathrm{~mm}$ con la pipeta modificada $(\mathrm{p}<0.05)$. Se concluye que al utilizar la pipeta modificada es factible depositar un fluido más cercanamente al útero.

Palabras clave: cateterización, cérvix, oveja, pipeta de inseminación

\section{AbSTRACT}

The aim of this study was to compare the depth of penetration of a dye into the cervical canal in sheep, simulating an insemination by using a traditional artificial insemination (AI) gun and one with a special design that has at the end an inflatable balloon that prevents reflux of dye. Eighty reproductive tracts of non-pregnant ewes

\footnotetext{
${ }^{1}$ Laboratorio Central, Facultad de Ciencias Agropecuarias, Universidad Alas Peruanas, Pachacamac, Lima, Perú

2 Departamento de Producción, Camal de Yerbateros, Lima, Perú

${ }^{3}$ E-mail: rodriguezgavancho@hotmail.com
}

Recibido: 13 de junio de 2016

Aceptado para publicación: 15 de octubre de 2016 
were collected from the local slaughterhouse in Lima, Peru and randomly distributed for catheterization with one of the two AI guns, depositing $0.5 \mathrm{ml}$ of methylene blue. The balloon of the second AI gun was inflated before depositing the die. Subsequently, the cervical canal was exposed and the depth of entry of the AI guns and the dye were measured considering the edge of the first cervical ring as zero $(0)$. Both AI guns penetrated $19.9 \pm 9.6$. The die reached the same depth with the traditional gun and $60.0 \pm 9.7 \mathrm{~mm}$ with the modified pipette $(\mathrm{p}<0.05)$. It is concluded that by using the modified pipette is possible to deposit a fluid more closely to the uterus.

Key words: catheterization, cervix, sheep, insemination pipette

\section{INTRODUCCIÓN}

Una de las herramientas más eficientes y económicas para el mejoramiento genético en las explotaciones pecuarias es la inseminación artificial (IA). En la oveja, la técnica de IA más utilizada es a través de la vía vaginal, utilizando semen fresco, lográndose tasas de fertilidad de entre 40 y $60 \%$ (Anel et al., 2006). La IA con semen congelado no está muy difundida en la industria ovina, debido principalmente a las bajas tasas de fertilidad obtenidas, posiblemente por el daño espermático que ocurre durante el proceso de criopreservación (Ruiz et al., 2015; Santiani et al., 2007).

El pasaje de la pipeta de inseminación a través del canal cervical del ovino es mucho más complicado que en el caso del bovino, pues es un canal cervical estrecho, sinuoso y con fondos de saco ciego con alrededor de cinco a siete pliegues cervicales excéntricos, de allí que el semen se deposita en la entrada del canal cervical (Kershaw et al., 2005).

Existe una variedad de técnicas diseñadas para lograr el pasaje de la pipeta de IA a través del canal cervical para depositar el semen cerca o dentro del útero. En la inseminación transcervical desarrollada en $\mathrm{Ca}$ nadá (Guelph system for transcervical AI) se coloca a la oveja en posición decúbito dorsal y con ayuda de un espéculo y una fuente de luz se logra penetrar, bajo ciertas condiciones, el canal cervical, mejorando las tasas de fertilidad, tanto con semen fresco como con semen congelado (Halbert et al., 1990; Buckrell et al., 1994; Winzor et al., 1994). Asimismo, se dispone de otro tipo de pipeta para inseminar los animales en pie (WulsterRadcliffe et al., 2002, 2004), así como técnica más complejas y costosas que incluyen el uso de dilatadores de cérvix (Sayre y Lewis, 1997; Bartlewski y Candappa, 2015; Falchi y Scaramuzzi, 2015).

En las grandes explotaciones ovejeras, las puntas o rebaños de empadre están conformadas por cientos de ovejas, y los programas de IA se llevan a cabo en las primeras semanas de la campaña de empadre, inseminándose hasta más de 100 ovejas por día, de modo que se requiere de métodos y procedimientos que permitan trabajar con rapidez y que sean económicos.

El presente estudio tuvo como objetivo comparar la profundidad de penetración de un colorante en el canal cervical de la oveja, simulando una inseminación con una pipeta de uso tradicional con otra de diseño especial, que dispone en el extremo un globo inflable que impide el reflujo del colorante.

\section{Materiales y Métodos}

En el camal de Yerbateros, de El Agustino, Lima, se recuperaron 80 tractos reproductivos de ovejas vacías de condición corporal regular. Los tractos se colocaron en 
suero fisiológico a $30{ }^{\circ} \mathrm{C}$ y se transportaron en cajas térmicas al Laboratorio Central de la Universidad Alas Peruanas (Pachacamac, Lima), donde se retiraron los tejidos adyacentes al aparato reproductor, incluyendo parte de la vagina. Se determinó la longitud del cuello uterino, considerando sus características externas. Los tractos reproductivos se distribuyeron al azar en dos grupos.

Las ovejas eran mayormente criollas, de más de dos dientes, y se encontraban dentro del periodo correspondiente al empadre de las ovejas en el Perú. Se usaron aparatos genitales que presentaban flujo vaginal y cuyos ovarios presentaban folículos, descartando aquellos que no cumplían con estos criterios o que presentaban cuerpo lúteo.

Se utilizaron dos tipos de pipetas para la cateterización transcervical: la pipeta de inseminación artificial tradicional, cuyo extremo libre está aguzada con una pequeña curvatura, la cual se viene utilizando desde los años 70 en trabajos de inseminación artificial en las zonas altoandinas de los departamentos de Pasco y Junín, y un modelo de pipeta modificado cuyo extremo libre presenta un pequeño globo para inflar, tipo sonda Foley de dos vías (Figura 1). Esta pipeta fue preparada a base de una sonda Foley N. ${ }^{\circ} 8$, recortada en $0.5 \mathrm{~cm}$ en el extremo libre, pero manteniendo el balón inflable, e interiormente, se colocó a lo largo de la sonda, un tubo metálico del grosor de una aguja hipodérmica N. ${ }^{\circ} 18$. Además, se utilizó azul de metileno como colorante, una regla graduada de 20 $\mathrm{cm}$ de largo y una regla pie de rey.

Se simuló la inseminación artificial mediante el pasaje del canal cervical con las pipetas en estudio. Para esto, la cérvix se sostuvo con una mano mientras con la otra se introducía la pipeta en el canal cervical, tratando de llegar lo más profundo posible. Allí se depositó $0.5 \mathrm{ml}$ de azul de metileno (como semen). En el caso de la pipeta modificada, un ayudante inflaba el globo de la pipeta con $5 \mathrm{ml}$ de agua destilada. Luego, los tractos reproductivos (cérvix y parte del úte- ro) fueron abiertos y se midió la distancia que alcanzó penetrar el colorante (Figura 2).

\section{Resultados y Discusión}

La profundidad de penetración dentro del canal cervical de las dos pipetas de inseminación fue de 19.9 $\pm 9.6 \mathrm{~mm}$ (rango de 4 a $33 \mathrm{~mm}$ ). Estos resultados difieren de los reportados por Kerchaw et al. (2005), aunque bastantes similares a los obtenidos por Falchi et al. (2012) en ovejas Ile-de-France, Romanov, Vendeenne y Sarda, y con el trabajo de Alvarez et al. (2012) en ovejas Churra y Assaf. Las diferencias, en algunos casos mínimas deben, no obstante, ser tomadas con cautela, pues el presente estudio se hizo con tractos reproductivos obtenidos en el camal y los otros estudios fueron realizados en animales vivos.

La profundidad de penetración del colorante fue diferente entre los dos tipos de pipeta $(\mathrm{p}<0.05)$. El colorante no hizo mayor progreso cuando se empleó la pipeta tradicional, lo cual es explicable por la presencia de los pliegues cervicales que fuerza el reflujo del líquido, mientras que en el grupo con la pipeta modificada se alcanzó una profundidad de penetración del colorante de $60.0 \pm$ $9.7 \mathrm{~mm}$ (rango de 41 a $78 \mathrm{~mm}$ ); es decir, dos veces más que la profundidad de penetración alcanzada por la pipeta. Este resultado es corroborado por Álvarez et al. (2012), quienes indican que cuando no se alcanza una penetración mayor de $30 \mathrm{~mm}$, se observa un reflujo total o parcial del semen hacia el canal vaginal. En el caso del estudio, el globo inflado de la pipeta dentro del canal cervical impidió el reflujo del colorante hacia el canal vaginal.

\section{Conclusiones}

La pipeta modificada en trabajos de inseminación cervical podría mejorar el depósito de semen cerca o dentro del útero en ovejas en estro. 


\section{Pipeta de uso tradicional}

\section{Pipeta de diseño especial}

Figura 1. Vista de las pipetas de inseminación artificial

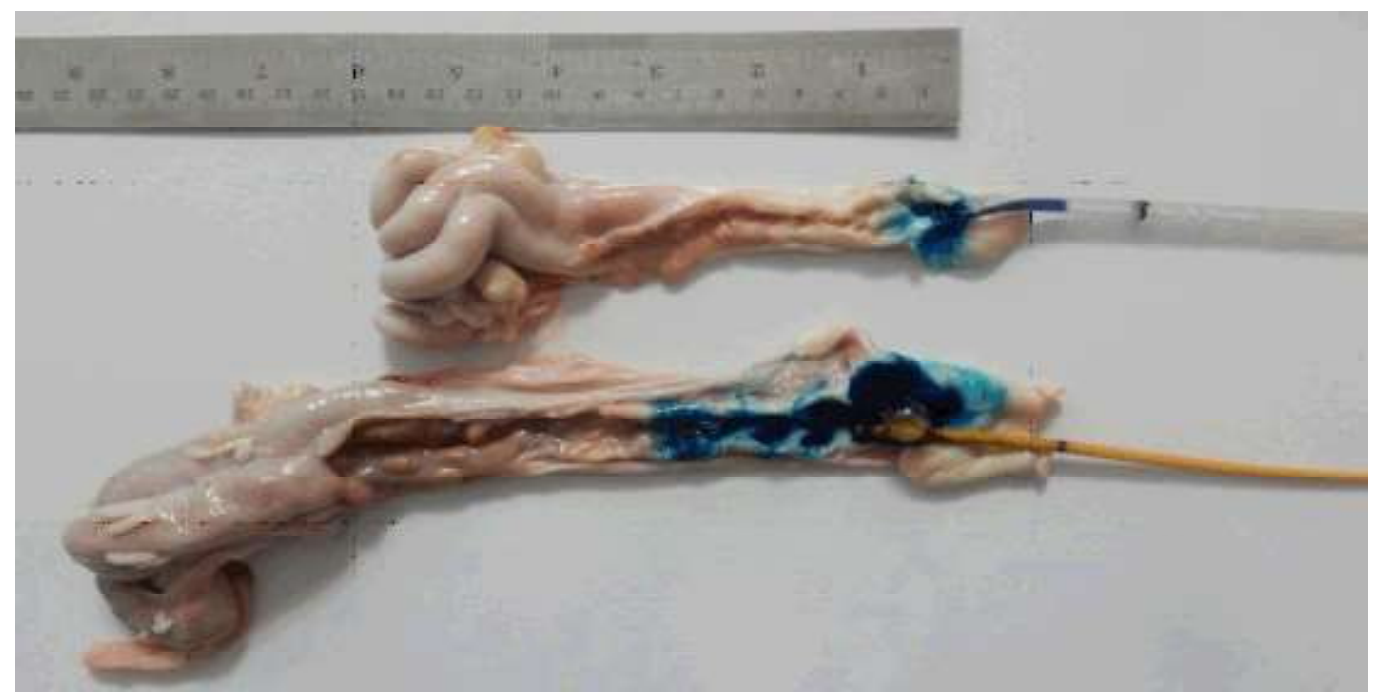

Figura 2. Comparación del alcance obtenido del colorante según la pipeta de inseminación artificial empleada.

\section{Literatura Citada}

1. Álvarez M, Chamorro C, Kaabi M, Anel-Lopez, E, Boixo J, Anel E, Anel L, De Paz P. 2012. Design and in vivo evaluation of two adapted catheters for intrauterine transcervical insemination in sheep. Anim Reprod Sci 131: 153-159. doi: 10.1016/j.anirepro-sci.2012.03.001

2. Anel L, Alvarez M, Martinez-Pastor F, Garcia-Macias V, Ane E, dePaz P. 2006. Improvement strategies in ovine artificial insemination. Reprod Domest Anim 41: 30-42. doi: 10.1111/j. 14390531.2006. 00767.x 
3. Bartlewski PM, Candappa I. 2015. Assessing the usefulness of prostaglandin $\mathrm{E}_{2}$ (Cervidil) for transcervival artificial insemination in ewes. Theriogenology 84: 1594-1602. doi: 10.1016/j.theriogenology. 2015. 08.007

4. Buckrell BC, Buschbeck C, Gartley CJ, Kroetsch T, McCutcheon W, Martin J, et al. 1994. Further development of a transcervical technique for artificial insemination in sheep using previously frozen semen. Theriogenology 42: 601-611. doi: 10.1016/0093691X(94)90377-U

5. Falchi L, Taema M, La Clanche S, Scaramuzzi R. 2012. The pattern of cervical penetration and the effect of topical treatment with prostaglandin and/ or FSH and oxytocin on the depth of cervical penetration in the ewe during the peri-ovulatory period. Theriogenology 78: 376-384. doi: 10.1016/j.theriogenology. 2012.02.017

6. Falchi L, Scaramuzzi R. 2015. An in vitro investigation of the actions of reproductive hormones on the cervix of the ewe in the follicular stage: the effects of 17â-estradiol, oxytocin, FSH, and arachidonic acid on the cervical pathway for the synthesis of prostaglandin E2. Theriogenology 83: 1007-1014. doi: 10.1016/j.theriogenology.2014.12.003

7. Halbert GW, Dobson H, Walton JS, Buckrell BC. 1990. A technique for transcervical intrauterine insemination of ewes. Theriogenology 33: 993-1010. doi: 10.1016/0093-691X(90)90061-W

8. Kershaw C, Khalid M, McGowan M, Ingram K, Leethongdee S, Wax G, Scaramuzzi R. 2005. The anatomy of the sheep cervix and its influence on the transcervical passage of an inseminating pipette into the uterine lumen. Theriogenology 64: 1225-1235. doi: 10.1016/j.theriogenology.2005.02.017
9. Ruiz L, Sandoval R, Santiani A. 2015. Evaluación de la calidad espermática del semen ovino posdescongelación al emplear dos fuentes energéticas y dos crioprotectores. Rev Inv Vet Perú 26: 4956. doi: 10.15381/rivep.v26i1.10942

10. Santiani A, Ruiz L, Sandoval R, Evangelista S, Urviola M, Catacora $N$, et al. 2007. Incremento de la tasa de no retorno de celo en ovejas utilizando un antioxidante análogo de superóxido dismutasa (Tempo) durante la criopreservación de semen. En: XX Reunión de la Asociación Latinoamericana de Producción Animal. Cusco, Perú.

11. Sayre B, Lewis G 1997. Fertility and ovum fertilization rate after laparoscopic or transcervical intrauterine artificial insemination oxytocin-treated ewes Theriogenology 48: 267-275.

12. Windsor D, Széll A, Buschbeck $C$, Edward A, Milton J, Buckrell BC. 1994. Transcervical artificial insemination of Australian Merino ewes with frozen-thawed semen. Theriogenology 42: 147-157. doi: 10.1016/0093691X(94)90671-5

13. Wulster-Radcliffe MC, Lewis GS. 2002. Development of a new transcervical artificial insemination method for sheep: effects of a new transcervical artificial insemination catheter and traversing the cervix on semen quality and fertility. Theriogenology 58: 13611371. doi: 10.1016/S0093-691X(02)01042-7

14. Wulster-Radcliffe, MC, Wang S, Lewis G. 2004. Transcervical artificial insemination in sheep: effects of a new transcervical artificial insemination instrument and traversing the cervix on pregnancy and lambing rate. Theriogenology 62: 990-1002. doi: 10.1016/ j.theriogenology.2003.12.031 